\title{
The expression and clinical significance of ZBTB7 in transitional cell carcinoma of the bladder
}

\author{
CHANGCHENG GUO*, DABO XIONG* , BIN YANG, HAIMING ZHANG, WENYU GU, \\ MIN LIU, XUDONG YAO, JUNHUA ZHENG and BO PENG
}

\begin{abstract}
Department of Urology, Shanghai Tenth People's Hospital, Tongji University School of Medicine, Shanghai 200072, P.R. China
\end{abstract}

Received November 3, 2015; Accepted June 27, 2017

DOI: 10.3892/ol.2017.6814

\begin{abstract}
Zinc finger and BTB domain containing 7A (ZBTB7) is a ZBTB protein family member of transcriptional repressors that serves a critical role in cell transformation and malignancy. However, the association between ZBTB7 expression in bladder cancer tissues and the prognosis of patients remains unclear. The aim of the current study was to detect the expression of ZBTB7 in transitional cell carcinoma (TCC) of the bladder and normal bladder mucous tissues to evaluate the diagnostic and prognostic value of ZBTB7 in TCC of the bladder. A total of 100 TCC specimens were analyzed and the expression of ZBTB7 mRNA was examined via reverse transcription-quantitative polymerase chain reaction. The expression of ZBTB7 protein was examined by western blotting and immunohistochemistry. The association between ZBTB7 expression and the clinical prognosis of patients from the TCGA database was analyzed. High expression of ZBTB7 mRNA and protein in TCC tissue was detected and TCC expression was significantly higher in TCC tissue than in normal bladder mucous tissues $(\mathrm{P}<0.05)$. Furthermore, ZBTB7 expression was associated with recurrence, a larger tumor size and higher tumor grade. In terms of overall and recurrence-free survival, the group expressing high levels of ZBTB7 exhibited lower overall and recurrence-free survival compared with the low ZBTB7 expression group, although these differences were not statistically significant. Therefore, ZBTB7 may be important in the initiation and progression of TCC.
\end{abstract}

Correspondence to: Dr Bo Peng or Dr Junhua Zheng, Department of Urology, Shanghai Tenth People's Hospital, Tongji University School of Medicine, 301 Yanchang Road, Shanghai 200072, P.R. China

E-mail: pengbo6908@163.com

E-mail: zhengjh0471@sina.com

${ }^{*}$ Contributed equally

Key words: ZBTB7, transitional cell carcinoma of the bladder, prognosis, survival

\section{Introduction}

Bladder cancer $(\mathrm{BC})$ is the fifth most common cancer and the second most diagnosed genitourinary cancer in Western countries (1). In China, BC is the leading cause of mortality of all urinary malignancies $(2,3)$. $\mathrm{BC}$ may be non-muscle invasive or muscle invasive. Patients with non-muscle invasive $\mathrm{BC}$ have a high risk of experiencing tumor recurrence and progression into muscle invasive $\mathrm{BC}$ and the prognosis of patients with muscle invasive $\mathrm{BC}$ is poor due to its high rate of metastasis (4). It has previously been demonstrated that the epithelial-mesenchymal transition (EMT) is important in BC (5). However, the precise mechanism of how the EMT regulates BC invasion remains unclear.

Zinc finger and BTB domain containing 7A (ZBTB7) is a ZBTB protein family member that critically influences cellular differentiation in a pleiotropic manner (6). ZBTB7 consists of four $\mathrm{COOH}$-terminal krüppel-type zinc fingers and an NH2-terminal POZ/BTB domain (6). The POZ/BTB domain is involved in homodimerization and heterodimerization and recruits corepressors including B-cell lymphoma 6 protein (BCL-6) and nuclear receptor corepressors 1 and 2. The krüppel-type zinc finger domain mediates specific DNA recognition and binding (7). ZBTB7 is overexpressed in different types of human cancer, including non-small cell lung, prostate, ovarian and breast carcinoma, as well as glioma, T-cell and B-cell lymphoma (8-13). A previous study by the current authors suggested that the expression of E-cadherin in bladder cancer cells may be inhibited by ZBTB7 and that low E-cadherin expression may alter the phenotype and apical-base polarity of epithelial cells in the T24 cell line (4). Therefore, ZBTB7 may regulate the EMT in bladder cancer cells. However, the levels of ZBTB7 expression in bladder cancer tissues and how ZBTB7 regulates EMT remain unknown.

The present study investigated the expression of ZBTB7 in transitional cell carcinoma of the bladder (TCC). The expression of ZBTB7 in TCC and normal human bladder tissues was evaluated using immunohistochemistry, reverse transcription-quantitative polymerase chain reaction (RT-qPCR) and western blotting to investigate whether ZBTB7 expression was associated with the clinicopathological characteristics and prognosis of patients with TCC. 


\section{Patients and methods}

Patients. Between September 2008 and June 2014, 100 patients (72 males and 28 females; mean age 66.3) pathologically diagnosed with TCC were enrolled in the current study at the Department of Shanghai Tenth People's Hospital, Tongji University (Shanghai, China). Pre-operative clinical data for each patient, including complete blood count, liver biochemistry, renal function, and tumor size were assembled in a computerized database. Two different types of tissues from each patient with TCC, including tissue taken from the TCC tumor itself and tumor-free tissue taken $>5 \mathrm{~cm}$ from the tumor edge, were collected immediately following surgical resection. All tissue samples included the mucous and muscular layers. Areas of hemorrhage and tissue necrosis were excluded. Specimens from each patient were divided into two sections. One section was snap-frozen immediately following resection and stored in liquid nitrogen until required. The other section was preserved in $10 \%$ formaldehyde solution (at $62^{\circ} \mathrm{C}$ for $60 \mathrm{~min}$ ) and paraffin-embedded. The current study was performed following a protocol approved by the Ethics Committee of Shanghai Tenth People's Hospital, Tongji University School of Medicine (Shanghai, China). Written informed consent for participation was obtained from each patient.

$R T-q P C R$. ZBTB7 primers were designed using Primer 3 (http://bioinfo.ut.ee/primer3-0.4.0/primer3/) according to the sequence of ZBTB7 taken from Genbank (https:// www.ncbi.nlm.nih.gov/nuccore). $\beta$-actin acted as an internal control and its primers were synthesized by Shanghai Sangon Biological Engineering Technology \& Services Co., Ltd. (Shanghai, China). The primer sequences were as follows: $\beta$-actin forward, 5'-TGAAGGTGACAGCAGTCGGTT-3' and reverse, 5'-AGAAGTGGGGTGGCTTTTAGGA-3'; ZBTB7 forward, 5'-TTCACCAGGCAGGACAAG-3' and reverse, 5'-GGTTCTTCAGGTCGTAGTTG-3'.

Total RNA was isolated from the tissues using a Tiangen RNA isolation kit (Tiangen, Biotech, Co., Ltd., Beijing, China), following the manufacturer's protocol. The density of the bands was measured using a UV spectrophotometer. Total RNA was reverse-transcribed into cDNA using Takara PrimeScript ${ }^{\mathrm{TM}}$ RT reagent kit (Takara Bio, Inc., Otsu, Japan) following the manufacturer's protocol. qPCR was subsequently performed in triplicate for each sample using a SYBR ${ }^{\circledast}$ ExScript Real-time PCR kit (Takara Biotechnology Co., Ltd., Dalian, China). A $20 \mu 1$ reaction mixture was used, containing $2 \mu 1$ template DNA, $1 \mu 1$ primers, $10 \mu 1 \mathrm{SYBR}$ premix and $7 \mu 1 \mathrm{ddH}_{2} \mathrm{O}$. PCR was performed using a 7900HT Fast Real-Time PCR machine (Applied Biosystems; Thermo Fisher Scientific, Inc., Waltham, MA, USA) under the following conditions: $95^{\circ} \mathrm{C}$ for $30 \mathrm{sec}$, 40 cycles of $95^{\circ} \mathrm{C}$ for $5 \mathrm{sec}$ and $60^{\circ} \mathrm{C}$ for $30 \mathrm{sec}$. PCR results were quantified using the $-2^{\Delta \Delta \mathrm{Cq}}$ method (14).

Western blotting. Total protein was extracted using radioimmunoprecipitation assay lysis buffer (Sangon Biotech Co., Ltd., Shanghai, China). Protein concentration was determined using the bicinchoninic acid protein assay kit (Pierce; Thermo Fisher Scientific, Inc.). Equal quantities of protein $(30 \mu \mathrm{g})$ were separated by $8 \%$ sodium dodecyl sulphate-polyacrylamide gel electrophoresis with Tris-glycine running buffer and were subsequently transferred to nitrocellulose membranes. Membranes were blocked with $0.1 \%$ Tween in Tris-buffered saline for $60 \mathrm{~min}$ at room temperature and incubated with monoclonal antibodies against ZBTB7 (cat. no. ab70208) (1:200 dilution) and glyceraldehyde 3-phosphate dehydrogenase [GAPDH; (cat. no. ab8245); 1:500 dilution, both from Abcam, Cambridge, UK] at $4^{\circ} \mathrm{C}$ overnight. GAPDH acted as a control. The membranes were washed with PBS-0.05\% Tween-20 and then incubated with appropriate goat-anti-rabbit peroxidase-conjugated secondary antibody (cat. no. A0545; 1:1,000) or goat-anti-mouse peroxidase-conjugated secondary antibody (cat. no. A9044; 1:5,000) (both from Sigma-Aldrich; Merck KGaA, Darmstadt, Germany) for $1 \mathrm{~h}$ at $37^{\circ} \mathrm{C}$, washed again and developed using an enhanced chemiluminescence (ECL) western blotting system (Bio-Rad Laboratories, Inc., Hercules, CA, USA).

Immunohistochemical staining. Sections $(5 \mu \mathrm{m})$ were stained using the Sp method (15) using the Vectorstain Elite ABC kit (Vector Laboratories, Inc., Burlingame, CA, USA) following the manufacturer's protocol, and underwent high temperature and pressure antigen retrieval (the sections were immersed in $0.01 \mathrm{M}$ citric acid salt retrieval solution, $\mathrm{pH} 6.0$ for $10 \mathrm{~min}$ ) and were blocked with $2 \%$ goat serum (Gibco; Thermo Fisher Scientific, Inc.) for $20 \mathrm{~min}$ at room temperature. Following the addition of $50 \mu 1$ primary antibody against ZBTB7 (cat. no. ab70208; dilution, 1:100; Abcam), the sections were incubated at $4^{\circ} \mathrm{C}$ overnight. Negative controls were established using phosphate buffered saline instead of primary antibody. Subsequently, $50 \mu 1$ secondary antibody (Vectorstain Elite ABC kit; dilution, 1:100; Vector Laboratories, Inc., Burlingame, CA, USA) was added to the sections, which were incubated for $30 \mathrm{~min}$ at room temperature. Staining was performed using diaminobenzidine for $5 \mathrm{~min}$ at room temperature followed by counterstaining with hematoxylin for $1 \mathrm{~min}$ at room temperature. Following dehydration, sections were fixed using a graded ethanol series (70\% ethanol $5 \mathrm{~min}, 80 \%$ ethanol $5 \mathrm{~min}, 90 \%$ ethanol $5 \mathrm{~min}, 100 \%$ ethanol $5 \mathrm{~min}$ ) then treat with xylene for 10 min at room temperature and mounted with Permount ${ }^{\mathrm{TM}}$ mounting medium (Thermo Fisher Scientific, Inc.).

The slides were observed using a light microscope under five random high-power fields and 100 cells were counted in each field. The number of ZBTB7-positive cells with brownish granular staining in the nucleus and cytoplasm were estimated. The observer was blinded to the study and analysis was based upon the percentage of positively stained cells and the degree of staining. Positively stained cells were scored as follows: $<5 \%$ staining, $0 ; 6-25,1 ; 26-50,2 ; 51-75,3$ and $>75 \%$, 4 . Cells were also given a score according to the degree of the staining exhibited: Light yellow staining, 1; yellow, 2 and brown, 3 . A positive reaction was determined if the product of the two scores was $>1$ (16).

The cancer genome atlas (TCGA) database analysis. The TCGA database (http://cancergenome.nih.gov) was analyzed using the UCSC Cancer Genome Brower (https:// genome-cancer.soe.ucsc.edu/). These data included gene expression data from 436 patients with bladder cancer, which facilitated the further investigation of the overall and 
Table I. Clinicopathological characteristics of patients with TCC.

\begin{tabular}{|c|c|c|c|c|c|c|c|c|c|}
\hline \multirow[b]{2}{*}{ Variables } & \multirow[b]{2}{*}{ Patients } & \multicolumn{3}{|c|}{ Grade } & \multicolumn{3}{|c|}{ TNM stage } & \multirow[b]{2}{*}{ Primary carcinoma } & \multirow[b]{2}{*}{ Recurrent } \\
\hline & & Potential & Low grade & High grade & $\mathrm{T} 1$ & $\mathrm{~T} 2$ & $\mathrm{~T} 3$ & & \\
\hline Total & 100 & 24 & 36 & 40 & 40 & 48 & 12 & 81 & 19 \\
\hline \multicolumn{10}{|l|}{ Sex } \\
\hline Male & 72 & 19 & 25 & 28 & 29 & 34 & 9 & 63 & 9 \\
\hline Female & 28 & 5 & 11 & 12 & 11 & 14 & 3 & 18 & 10 \\
\hline \multicolumn{10}{|l|}{ Age } \\
\hline$<45$ & 1 & 0 & 0 & 1 & 0 & 0 & 1 & 1 & 0 \\
\hline $45-65$ & 65 & 9 & 27 & 29 & 25 & 29 & 11 & 57 & 8 \\
\hline$>65$ & 34 & 15 & 9 & 10 & 15 & 19 & 0 & 23 & 11 \\
\hline
\end{tabular}

TCC, transitional cell carcinoma; TNM, tumor-node-metastasis.

A

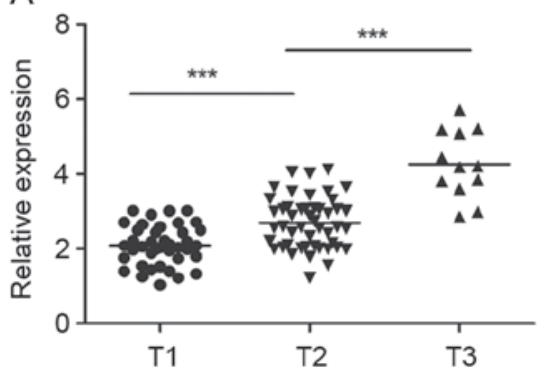

B

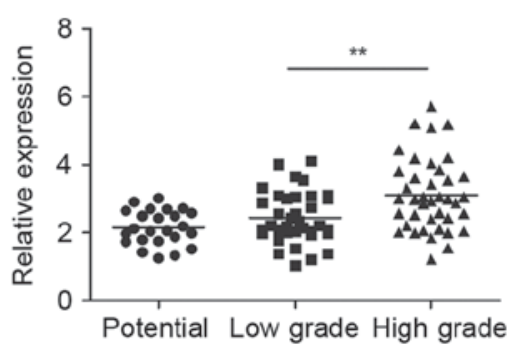

C

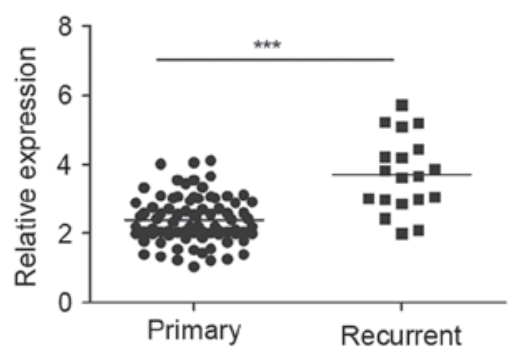

Figure 1. ZBTB7 expression in patients with TCC. (A) ZBTB7 expression in patients with T1, T2, T3 stage TCC. (B) ZBTB7 expression in potential, low and high grade TCC. (C) ZBTB7 expression in primary and recurrent TCC. TCC, transitional cell carcinoma; ZBTB7, zinc finger and BTB domain containing 7A. ${ }^{* *} \mathrm{P}<0.01,{ }^{* * *} \mathrm{P}<0.001$.

recurrence free survival in groups with low and high levels of ZBTB7 expression.

Statistical analysis. Statistical analysis was performed using the IBM SPSS software v20.0 (IBM Corp., Armonk, NY, USA). Student's t-test was used as statistical methods to compare the difference in ZBTB7 mRNA expression between normal bladder tissues and TCC tissues of different grades and stages, and between primary and recurrent cases. To compare the level of ZBTB7 protein expression (two group: Positive and negative group) between TCC tissues of different grades and stages, and between primary and recurrent cases, the $\chi^{2}$ test was used. The log-rank test was used to analyze the significant of low expression and high expression of ZBTB7. $\mathrm{P}<0.05$ was considered to indicate a statistically significant difference. Comparison of survival curves was conducted using the log-rank test.

\section{Results}

Clinicopathological characteristics of selected patients. A total of 100 patients with TCC, recruited from the Department of Urology, Shanghai Tenth People's Hospital between September 2010 and June 2014, were included in the present study. The clinicopathological characteristics of these patients are summarized in Table I.
The expression of ZBTB7 $m R N A$ in patients with TCC. The association between ZBTB7 mRNA expression in TCC samples and patient clinicopathological characteristics was analyzed (Fig. 1). Firstly, the association between tumor size, as determined by the tumor-node-metastasis (TNM) classification system was analyzed (17). The expression of ZBTB7 mRNA was significantly higher in larger than smaller tumors. The relative expression of ZBTB7 in T1 stage tumors was $2.08 \pm 0.53$, in T2 it $2.69 \pm 0.68$, significantly higher than in $\mathrm{T} 1$ stage tumors $(\mathrm{P}<0.01)$ and in T3 it was 4.26 \pm 0.90 , significantly higher than in T2 stage tumors $(\mathrm{P}<0.01$; Fig. 1A). ZBTB7 expression was significantly higher in high grade tumors compared with low-grade tumors $(\mathrm{P}<0.05$; Fig. 1B). However, there is no significant difference between the low and potential grade groups. In terms of grade, as determined by the WHO 1973 grading system and the new WHO classification of malignant tumors of the urinary tract 2004 (18), the relative expression of ZBTB7 was $2.15 \pm 0.51$ in potential grade tumors, $2.44 \pm 0.76$ in low grade tumors and $3.10 \pm 1.06$ in high grade tumors. Additionally, ZBTB7 expression was significantly higher in recurrent tumors $(3.70 \pm 1.09)$ compared with primary tumors $(2.39 \pm 0.69 ; \mathrm{P}<0.01$; Fig. 1C).

The expression of ZBTB7 protein in normal human bladder and TCC tissues. ZBTB7 protein was measured in the nucleus and cytoplasm. Following immunohistochemical staining, cells with high ZBTB7 expression were stained a brownish yellow 
Table II. The associations between ZBTB7 IHC and clinicopathological indicators.

\begin{tabular}{|c|c|c|c|c|c|}
\hline Pathological feature & Number & Positive & Negative & Rate of expression, $\%$ & P-values ${ }^{\mathrm{a}}$ \\
\hline \multicolumn{6}{|l|}{ TNM stage } \\
\hline $\mathrm{T} 1$ & 40 & 28 & 12 & 70 & \multirow{3}{*}{0.424} \\
\hline $\mathrm{T} 2$ & 32 & 28 & 4 & 87.50 & \\
\hline $\mathrm{T} 3$ & 28 & 20 & 8 & 71.43 & \\
\hline \multicolumn{6}{|l|}{ Grade } \\
\hline Potential grade & 12 & 2 & 10 & 16.60 & \multirow{3}{*}{0.003} \\
\hline Low grade & 48 & 40 & 8 & 83.33 & \\
\hline High grade & 40 & 32 & 8 & 80 & \\
\hline \multicolumn{6}{|l|}{ Primary or recurrent } \\
\hline Primary & 19 & 19 & 0 & 100 & \\
\hline Recurrent & 81 & 60 & 19 & 75 & 0.185 \\
\hline
\end{tabular}

A

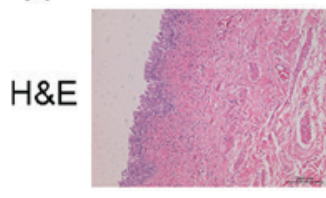

IHC

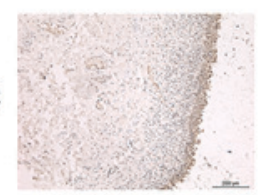

B

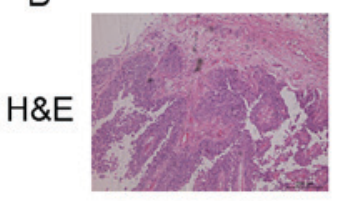

$\mathrm{IHC}$

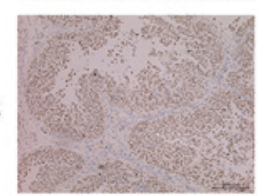

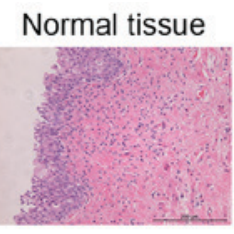

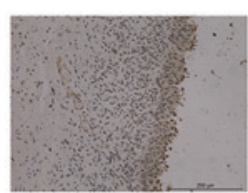

Tumor tissue
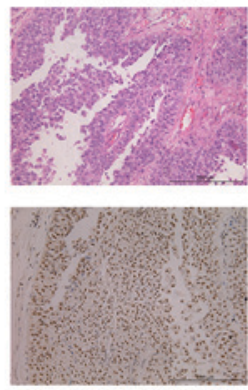
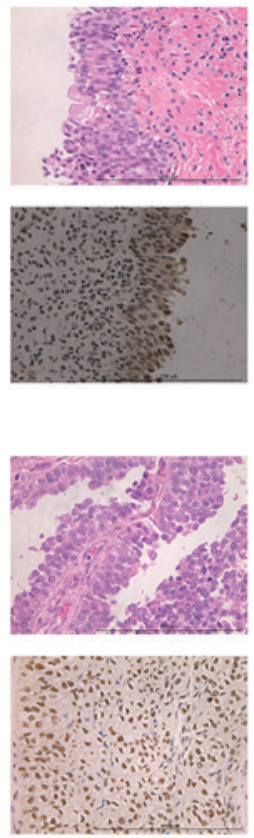

Figure 2. H\&E and IHC staining for ZBTB7 in normal and tumor tissue. (A) H\&E and IHC staining for ZBTB7 in normal tissue. ZBTB7 staining was positive only in superficial mucous layer. (B) H\&E and IHC staining for ZBTB7 in tumor tissue. ZBTB7 staining was positive throughout the mucous and muscular layers. Left panels, x100 magnification; middle panels, x200 magnification; right panel, x400 magnification. H\&E, hematoxylin and eosin; IHC, immunohistochemistry; ZBTB7, zinc finger and BTB domain containing 7A.

granular color (Fig. 2). The expression of ZBTB7 protein in normal bladder tissue was positive only in the mucous layer. However, in TCC tumor tissue, the mucous and muscular layer exhibited positive ZBTB7 expression. The positive rate of ZBTB7 protein expression in TCC tumors of different stages and grades were as follows (Table II): T1, 70\%; T2, 87.5\%; T3, $83.3 \%$; potential grade, $16.6 \%$; low grade, $83.33 \%$; high grade, $80 \%$; recurrent tumor, $100 \%$ and primary tumor, $75 \%$. The expression of ZBTB7 protein differed significantly between TCC and normal bladder tissues $(\mathrm{P}<0.05)$ (Fig. 2) and between

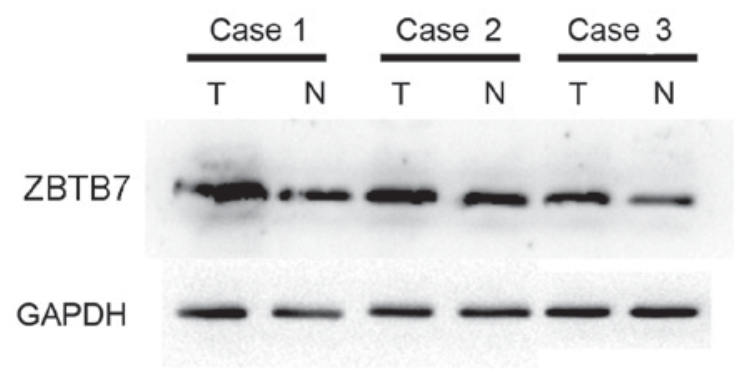

Figure 3. Western blot analysis of levels of ZBTB7 expression in three patients with transitional cell carcinoma. The expression of ZBTB7 protein is markedly increased in tumor tissue. T, transitional cell carcinoma samples; $\mathrm{N}$, normal samples; GAPDH, glyceraldehyde 3-phosphate dehydrogenase; ZBTB7, zinc finger and BTB domain containing 7A.

tumors of different TCC grades (potential grade vs. low grade, $\mathrm{P}<0.05$ ) (Table II). The associations between the expression of ZBTB7 protein in TCC tissues and patient clinicopathological indicators are presented in Table II. No significant differences were found between tumors in different TNM stages $(\mathrm{P}>0.05)$, however ZBTB7 protein expression differed significantly among tumors with different histological grades (potential grade vs. low grade, $\mathrm{P}<0.05)$.

Levels of ZBTB7 protein in TCC bladder and normal tissue were detected by western blot analysis and it was observed that the expression of ZBTB7 protein was markedly higher in tumor tissue than normal tissue (Fig. 3).

ZBTB7 gene expression is not associated with the clinical prognosis of TCC. Information from 436 patients with $\mathrm{BC}$ patients from the TCGA database were analyzed and patients with higher ZBTB7 expression exhibited a lower overall survival rates compared with patients with low ZBTB7 expression (Fig. 4A). However, this difference was not statistically significant $(\mathrm{P}=0.75)$. Recurrence free survival in patients with low expression of ZBTB7 compared with patients with high expression of ZBTB7 was also analyzed. Recurrence-free survival was generally lower in patients with high ZBTB7 expression. However, this difference was not significant $(\mathrm{P}=0.43)$. 
A

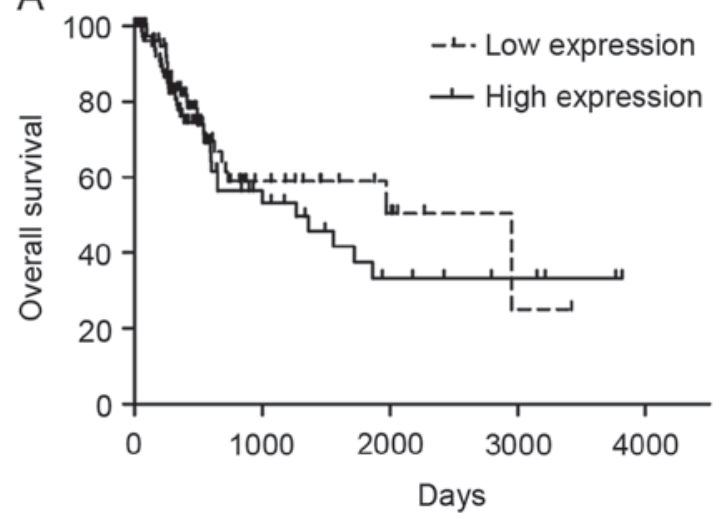

B

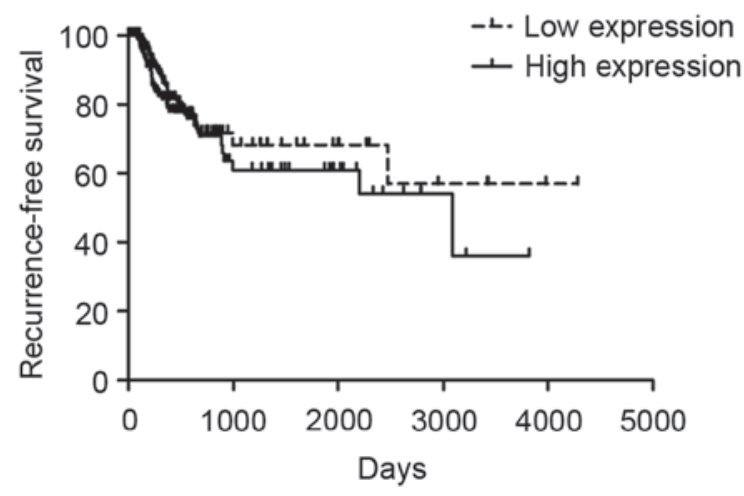

Figure 4. Associations between ZBTB7 expression and the clinical prognosis of patients with transitional cell carcinoma. (A) The overall survival curve indicated that patients with high expression of ZBTB7 exhibited lower overall survival levels compared with patients with low ZBTB7 expression. However, this difference between the two groups was not statistically significant $(\mathrm{P}>0.05)$. (B) The recurrence-free survival curve indicated that there was a decline in recurrence-free survival in patients exhibiting high expression of ZBTB7. However, there was still no significant difference between two groups (P>0.05). ZBTB7, zinc finger and BTB domain containing 7A.

\section{Discussion}

$\mathrm{BC}$ is responsible for 150,000 cases of cancer-associated mortality annually and is the fourth most common malignant neoplasm diagnosed in males worldwide (19). Approximately $70-80 \%$ cases of BCs are non-muscle invasive and the remaining $20-30 \%$ are muscle invasive at initial diagnosis. Between 30 and $50 \%$ of patients with non-muscle invasive $\mathrm{BC}$ experience recurrence following transurethral resection of the primary tumor and $10-20 \%$ progress to muscle invasive $\mathrm{BC}$ (4). Therefore, patients with non-muscle invasive $\mathrm{BC}$ have a high risk of experiencing recurrence and progression to muscle invasive BC. Despite therapeutic advancements in BC treatment, including surgery and neoadjuvant chemotherapy, there were 16,000 cases of BC-associated mortality in the USA in 2015 alone (20).

A number of potential markers for $\mathrm{BC}$ have been identified, including zinc finger protein 671 (21) and C-X-C motif chemokine ligand 1 (22). However, there are still no characterized, non-invasive molecular markers able to detect $\mathrm{BC}$ tumors and predict patient outcomes. Therefore, improved understanding of the molecular carcinogenesis of BC tumor progression may aid the development of novel, less invasive diagnostic markers. In the current study, high expression of ZBTB7 in BC tumor tissue was detected and it was determined that ZBTB7 expression may be associated with tumor size, grade and stage.

ZBTB7, a member of the ZBTB family, was identified by Maeda et al (6) in 2005. ZBTB7 is located at 19p13.3 and possesses two exons and two introns. It was initially considered to bind to HIV-1 promoter elements and was subsequently identified as a protein that interacts with BCL-6 and leads to the inactivation of mouse ZBTB7, inhibition of multicellular differentiation and embryonic lethality $(23,24)$. ZBTB7 is regarded as a master regulator as it binds specifically to p19 ARF, which is an important anti-oncogene. Chen et al (25) demonstrated that ZBTB7 is able to combine directly with the p19 ARF promoter and inactivate it in vivo. In addition, ZBTB7 is able to act upstream of many types of proto- and anti-oncogenes, which serve an important role in tumorigenesis and tumor biological behavior, whereas non-specific proto-oncogenes simply promote tumor cell growth (9).

Previous studies have indicated that ZBTB7 is highly expressed in different types of cancer, including lung and colon cancer $(6,8,11,26-28)$. In these neoplasms, it may promote tumorigenesis, acting as a proto-oncogene by repressing or enhancing the expression of genes involved in apoptosis, cell proliferation, differentiation and invasion. The present study demonstrated that ZBTB7 expression in TCC tissue was positive, indicating that ZBTB7 may promote the initiation and progression of TCC. By assessing the association between ZBTB7 gene expression in TCC and the clinicopathological features of patients, it was determined that the higher expression of ZBTB7 was associated with a more severe tumor TNM stage, tumor recurrence and a higher histological grade. These results are in concordance with previous studies that investigated the prognostic impact of ZBTB7 in TCC $(4,29)$. Therefore, high expression of ZBTB7 in TCC may be important in tumor initiation, progression and invasion.

The present study used the TCGA database to analyze the association between ZBTB7 expression and patient prognosis. Patients with high expression of ZBTB7 generally exhibited lower overall survival rates compared to those with low ZBTB7 expression. However, this difference was not statistically significant $(\mathrm{P}>0.05)$. This may be explained by two reasons. Firstly, no follow-up information existed on $>100$ patients out of the 436 patients included in the database. This loss of patients to follow-up may have skewed the end result. Secondly, the number of patients included in the current study was relatively small. Therefore, further studies involving larger cohorts are required to confirm the prognostic impact of ZBTB7 in TCC.

In conclusion, positive immunoreactivity of ZBTB7 is frequently observed in TCC and there was a significant association between high ZBTB7 expression and a higher tumor histological stage and grade. ZBTB7 expression may serve an important role in the initiation and progression of TCC and routine assessment of ZBTB7 expression may improve the identification of patients with high-risk TCC. 


\section{Acknowledgements}

This study was supported by the National Natural Science Foundation of China (grant no. 31100702/C100307) and Specialized Research Fund for the Doctoral Program of Higher Education in China (grant no. 20110072120054).

\section{References}

1. Siegel R, Ma J, Zou Z and Jemal A: Cancer statistics, 2014. CA Cancer J Clin 64: 9-29, 2014.

2. Jemal A, Bray F, Center MM, Ferlay J, Ward E and Forman D: Global cancer statistics. CA Cancer J Clin 61: 69-90, 2011.

3. Siegel R, Naishadham D and Jemal A: Cancer statistics, 2012. CA Cancer J Clin 62: 10-29, 2012.

4. Guo C, Zhu K, Sun W, Yang B, Gu W, Luo J, Peng B and Zheng J: The effect of ZBTB7 on bladder cancer epithelial-mesenchymal transition. Biochem Biophys Res Commun 443: 1226-1231, 2014.

5. Geng J, Fan J, Ouyang Q, Zhang X, Zhang X, Yu J, Xu Z, Li Q, Yao X, Liu X and Zheng J: Loss of PPM1A expression enhances invasion and the epithelial-to-mesenchymal transition in bladder cancer by activating the TGF- $\beta /$ Smad signaling pathway. Oncotarget 5: 5700-5711, 2014.

6. Maeda T, Hobbs RM, Merghoub T, Guernah I, Zelent A, Cordon-Cardo C, Teruya-Feldstein J and Pandolfi PP: Role of the proto-oncogene Pokemon in cellular transformation and ARF repression. Nature 433: 278-285, 2005.

7. Zhang QL, Tian DA and Xu XJ: Depletion of Pokemon gene inhibits hepatocellular carcinoma cell growth through inhibition of H-ras. Onkologie 34: 526-531, 2011.

8. Apostolopoulou K, Pateras IS, Evangelou K, Tsantoulis PK, Liontos M, Kittas C, Tiniakos DG, Kotsinas A, Cordon-Cardo C and Gorgoulis VG: Gene amplification is a relatively frequent event leading to ZBTB7A (Pokemon) overexpression in non-small cell lung cancer. J Pathol 213: 294-302, 2007.

9. Aggarwal A, Hunter WJ III, Aggarwal H, Silva ED, Davey MS, Murphy RF and AgrawalDK: Expression of leukemia/lymphomarelated factor (LRF/ZBTB7) in human breast carcinoma and other cancers. Exp Mol Pathol 89: 140-148, 2010.

10. Rovin RA and Winn R: Pokemon expression in malignant glioma: An application of bioinformatics methods. Neurosurg Focus 19: E8, 2005.

11. Qu H, Qu D, Chen F, Zhang Z, Liu B and Liu H: ZBTB7 overexpression contributes to malignancy in breast cancer. Cancer Invest 28: 672-678, 2010.

12. Maeda T, Merghoub T, Hobbs RM, Dong L, Maeda M, Zakrzewski J, van den Brink MR, Zelent A, Shigematsu H, Akashi K, et al: Regulation of B versus T lymphoid lineage fate decision by the proto-oncogene LRF. Science 316: 860-866, 2007.

13. Jiang L, Siu MK, Wong OG, Tam KF, Lam EW, Ngan HY, Le XF, Wong ES, Chan HY and Cheung AN: Overexpression of proto-oncogene FBI-1 activates membrane type 1-matrix metalloproteinase in association with adverse outcome in ovarian cancers. Mol Cancer 9: 318, 2010.

14. Livak KJ and Schmittgen TD: Analysis of relative gene expression data using real-time quantitative PCR and the 2(-Delta Delta C(T)) method. Methods 25: 402-408, 2001.
15. Shi SR, Key ME and Kalra KL: Antigen retrieval in formalinfixed, paraffin-embedded tissues: An enhancement method for immunohistochemical staining based on microwave oven heating of tissue sections. J Histochem Cytochem 39: 741-748, 1991.

16. Zhao ZH, Wang SF, Yu L, Wang J, Chang H, Yan WL, Fu K and Zhang J: Expression of transcription factor Pokemon in non-small cell lung cancer and its clinical significance. Chin Med J (Engl) 121: 445-449, 2008.

17. Sobin LH and Fleming ID: TNM classification of malignant tumors, fifth edition (1997). Union Internationale Contre le Cancer and the American Joint Committee on Cancer. Cancer 80: 1803-1804, 1997.

18. World Health Organization Classification of Tumours: Pathology and Genetics of Tumours of the Urinary Systemand Male Genital Organs. Eble JN, Sauter G, Epstein JI and Sesterhenn IA (eds). IARC Press, Lyon, 2004.

19. Chavan S, Bray F, Lortet-Tieulent J, Goodman M and Jemal A: International variations in bladder cancer incidence and mortality. Eur Urol 66: 59-73, 2014.

20. Scarpato KR, Morgans AK and Moses KA: Optimal management of muscle-invasive bladder cancer-a review. Res Rep Urol 7: 143-151, 2015.

21. Yeh CM, Chen PC, Hsieh HY, Jou YC, Lin CT, Tsai MH, Huang WY, Wang YT, Lin RI, Chen SS, et al: Methylomics analysis identifies ZNF671 as an epigenetically repressed novel tumor suppressor and a potential non-invasive biomarker for the detection of urothelial carcinoma. Oncotarget 6: 29555-29572, 2015.

22. Nakashima M, Matsui Y, Kobayashi T, Saito R, Hatahira S, Kawakami K, Nakamura E, Nishiyama H and Ogawa O: Urine CXCL1 as a biomarker for tumor detection and outcome prediction in bladder cancer. Cancer Biomark 15: 357-364, 2015.

23. Kelly KF and Daniel JM: POZ for effect-POZ-ZF transcription factors in cancer and development. Trends Cell Biol 16: 578-587, 2006.

24. Stogios PJ, Chen L and Privé GG: Crystal structure of the BTB domain from the LRF/ZBTB7 transcriptional regulator. Protein Sci 16: 336-342, 2007.

25. Chen WY, Zeng X, Carter MG, Morrell CN, Chiu Yen RW, Esteller M, Watkins DN, Herman JG, Mankowski JL and Baylin SB: Heterozygous disruption of Hicl predisposes mice to a gender-dependent spectrum of malignant tumors. Nat Genet 33: 197-202, 2003.

26. Zhao ZH, Wang SF, Yu L, Wang J, Chang H, Yan WL, Zhang J and Fu K: Overexpression of Pokemon in non-small cell lung cancer and foreshowing tumor biological behavior as well as clinical results. Lung Cancer 62: 113-119, 2008.

27. Jiao W, Liu F, Tang FZ, Lan J, Xiao RP, Chen XZ, Ye HL and Cai YL: Expression of the Pokemon proto-oncogene in nasopharyngeal carcinoma cell lines and tissues. Asian Pac J Cancer Prev 14: 6315-6319, 2013.

28. Zhao GT, Yang LJ, Li XX, Cui HL and Guo R: Expression of the proto-oncogene Pokemon in colorectal cancer-inhibitory effects of an siRNA. Asian Pac J Cancer Prev 14: 4999-5005, 2013.

29. Li W, Kidiyoor A, Hu Y, Guo C, Liu M, Yao X, Zhang Y, Peng B and Zheng J: Evaluation of transforming growth factor- $\beta 1$ suppress Pokemon/epithelial-mesenchymal transition expression in human bladder cancer cells. Tumour Biol 36: 1155-1162, 2015. 